\title{
Study of Electrocapillarity in Dielectrics Using Palm Oil
}

\author{
Nwodo A. $\mathbf{N}^{1}$, Ugwu E.I $\mathbf{I}^{2, *}$ \\ ${ }^{1}$ Department. of Physics, Federal University, Ndufu-Alike - Ikwo, \\ ${ }^{2}$ Department of Industrial Physics, Ebonyi State University, P.B.M 53, Abakaliki, Nigeria \\ *Corresponding author: ugwuei@yahoo.com
}

Received May 19, 2014; Revised June 07, 2014; Accepted June 20, 2014

\begin{abstract}
This paper presents analytical and experimental study of electrocapillarity in dielectrics under the frame work of parallel plates using palm oil.. First, Mathematical approach was used to derive an expression relating capillary ascent with the applied field. The results obtained from the experiment and that of the analytical results were compared with the expected results from the equation relating the liquid ascent and the applied field obtained from Lippmann equation, where it was observed that there is no evidence of linear relation between the height of the capillary rise of oil in the tube and the applied field.
\end{abstract}

Keywords: Electrocapillarity, Dielectric, parallel plates, Electric field, Palm oil, Capillary rise

Cite This Article: Nwodo A. N, and Ugwu E.I, "Study of Electrocapillarity in Dielectrics Using Palm Oil." International Journal of Physics, vol. 2, no. 3 (2014): 83-85. doi: 10.12691/ijp-2-3-3.

\section{Introduction}

Electrocappilary driven flow in a micro-structure is an important phenomena receiving tremendous attention in recent year since the advent of the study of surface tension which is known to be as a result of an inherent dominant force in micro scale. The theoretical and experimental investigation to unravel the concept has been reported in the literatures $[1,2,3]$. More attention was geared towards the study following the advent of scientist intention on the applicability and usefulness of microfludic coupled with the implication of the modification of surface tension by means of applying electric potential or temperature gradient as carried out and reported by Berge, Peseux and others who had investigated the use of electrocappilarity to change the of contact angle during capillary ascent by creating a transparent drop lens of variable focal length [4]. Prins et al demonstrated that the control of fluid motion by electrocapillarity pressure in three dimensional structures containing thousand of microchannels is more probable than use ordinary pressure. [5,6]. In accordance with various literatures, it is well known fact that the gravity is proportional to the cube of liquid droplet scale which is confirmed to invariably affect the capillary force on microfluidic flow and goes on dramatically to show that it even increases with decreasing scale thereby prompting many researchers to use numerical simulation to enhance the understanding of fluid flow behaviour in complex system. Especially numerical simulation based on LBM has been successfully applied in the study of wetting on spreading phenomena involving interfacial dynamics [7] and seem to have been much valuable method for studying capillary driven flow [8], apart from its applicability in simulation of electrowetting phenomenon [9]. This idea led delve into to look at the influence of change in contact angle of the droplets of liquid on a dielectric- covered conductive substrate [10] has been examined after it has been confirmed that increase in applied electric field does not affect contact angle as far as contact angle saturation has been reached which is $200 \mathrm{~V}$ [11]. In this work we intend to study how the applied electric field affect capillary rise of palm oil in a capillary tube through two parallel

\section{Theoretical Analysis}

The Schrodinger equation for molecular electrons of the palm oil is of the form

$$
\left[\hat{H}_{o}-i \hbar \partial / \partial t\right] \psi_{z}^{\prime}=e_{z} \sin \omega t \psi_{z}^{\prime} \exp \left(-l / \hbar E_{o} t\right)
$$

Neglecting the exponent with a common factor of $e^{i \omega t}$ and $e^{-i \omega t}$ which are linearly independent results from the solution of above equation to be of the form

$$
\begin{gathered}
H_{0} \omega_{+}-\left[E_{o}+\hbar \omega\right] \omega_{+}=\frac{e}{2 i} \psi_{o} \\
H_{o} \omega_{-}-\left[E_{o}-\hbar \omega\right] \omega_{-}=\frac{e}{2 i}
\end{gathered}
$$

As $E_{o}+\hbar \omega$ and $E_{o}-\hbar \omega$ are not eigenvalue of $\hat{H}$ since $\omega$ is not absorption frequency, the related eigen function can be expanded to be

$$
\omega_{+} \equiv \sum_{i=0}^{\infty} c_{i}^{\dagger} \psi_{i}
$$

This enables us to write that

$$
\hat{H}_{o} \psi_{i}=E_{i} \psi_{i}
$$


This invariably leads to

$$
h \omega_{i}=E_{i}-E_{o}
$$

Since the electric field component is said to be partially polarized in the molecules of oil, the field in operation here is from applied potential difference given as

$$
\gamma=\gamma_{o}-\frac{1}{2} c V^{2}
$$

In a pair of parallel metal plate as in the case of our experiment, the stored electrostatic energy in the polarized dielectric shows that surface tension, $\gamma$ of any liquid is related to electric field by

$$
\gamma=\gamma_{0}+\frac{1}{4} \varepsilon_{o}[\varepsilon-1] d E^{2}
$$

This is invariably the theoretical relation between surface tension and the applied potential difference across the plates which in terms of contact angle is given as

$$
\cos \theta-\cos \theta+\frac{1}{2} \frac{\varepsilon \varepsilon_{0}}{\gamma d} V
$$

Where $\mathrm{c}$ is the capacitance, and $\gamma, \gamma_{o}$ is the final and initial surface tension while $\mathrm{d}$ is the separation between the two parallel plates which contains the dielectric material. [2,12] Equations (1) is known as Lippmann equation while equations (5 and 7 ) were derived by combining the Young equation with the assumption that the change in surface tension at the sold/liquid interface is the energy stored in a capacitor across the dielectric. In terms of liquid ascent, equation (6) can be written as

$$
h-h_{o}=\frac{C V^{2}}{r \rho g}
$$

Since

$$
\begin{gathered}
E=\frac{V}{d} \\
h-h_{o}=\frac{C d}{r \rho g} E^{2}
\end{gathered}
$$

Considering the fact that a pair of parallel metal plates stored electrostatic energy in polarized dielectric, the liquid ascent can be written as

$$
h-h_{o}=\frac{1}{4} \varepsilon_{o}\left(\frac{\varepsilon-1}{\rho g r}\right) E^{2}
$$

From this theoretical framework deduction, non-liearity induced electric field term $C_{d}=-\frac{1}{2} \varepsilon_{0}(\varepsilon-1)$ is observed.

\section{Experimental Procedure}

The experiment was carried out by setting up a pair of horizontal metal plates measuring $14 \mathrm{~mm}$ x $10 \mathrm{~mm} 5 \mathrm{~mm}$ with $1 \mathrm{~mm}$ as distant of separation between them filled with Perspex as dielectric material. Two holes are made in between the center of the metal on top for the purposes of insertion of capillary tube and the other hole serves as an avenue through which air is expelled and also for pouring of palm oil as in Figure 1 Electrical contacts provided at the appropriate terminals enable connection to a highvoltage D.C source which read using a sensitive digital avommeter while the liquid ascent is read using the a traveling microscope at an interval as DC source is adjusted. The liquid is well filtered in order to limit an abrupt change in the liquid level in the capillary tube. As the experiment went on, the applied potential was carefully checked to avoid dielectric breakdown in the setup. The potential difference across the plates is read from the avometer while the liquid ascent is read using traveling microscope. The liquid is well filtered in order to reduce the probability of abrupt change in the liquid level in the capillary. This is because the maximum applied field in the experimental set-up is influenced by electrophoresis than dielectric breakdown

\section{Result and Discussion}

Figure one presents the graph of variation of liquid ascent in the capillary as a function of applied voltage for this experiment performed under ambient temperature from where it clearly obvious that the graph itself does not exhibit a linear as already observed by [12] relation accept within the threshold range which is identified between $0.00-50$ volt which led to maximum liquid ascent of $119 \times 10^{-2} \mathrm{~m}$. This according to theoretical report is expected due some unobservable factor such as change in the surface tension at the solid / liquid interface that often result from the alteration of liquid -solid interfacial energy as explained by Berge. [2,13] Invariably, it has also been observed that the applied potential tends not vary with contact angle a particular known as saturation potential. [14].

Figure 2 depicts the comparative analysis of the predicted and analytical results in relation to the applied potential and capillary rise using Young / Lippmann equation for surface tension while that of the logarithm of the applied field and the liquid ascent is shown in Figure 3 which did not show any significant indication of linearity of the system. However it was also observed that from this theoretical analysis there was no linear relation between the two variables as indicated in the predicted result. The analytical graph has the same values at only two points; $1.75 \times 10^{3} \mathrm{~V}$ and $3.20 \times 10^{3} \mathrm{~V}$. The observation to be noted in these results was that there is a required threshold voltage in order to obtain an appreciable liquid ascent during the experiment.

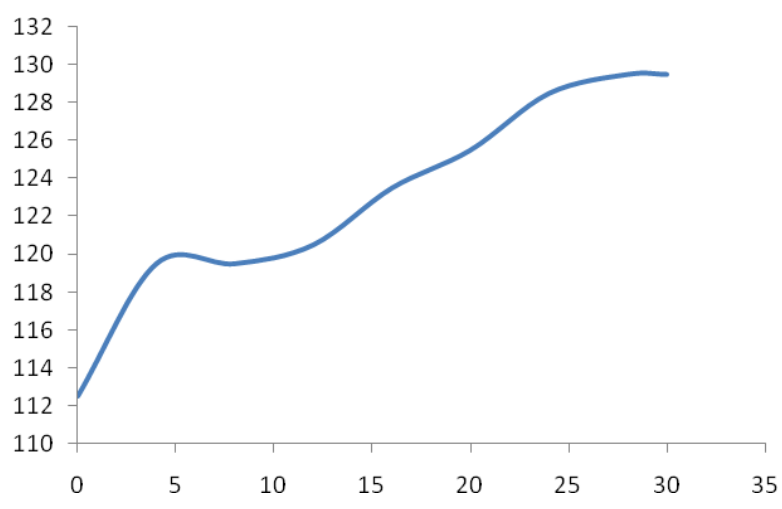

Figure 1. Graph of Liquid ascent $\mathrm{h} / 10^{-5} \mathrm{~m}$ as a of electric Field $\mathrm{E}_{\mathrm{x}} / 10 \mathrm{Vm}^{-1}$ 


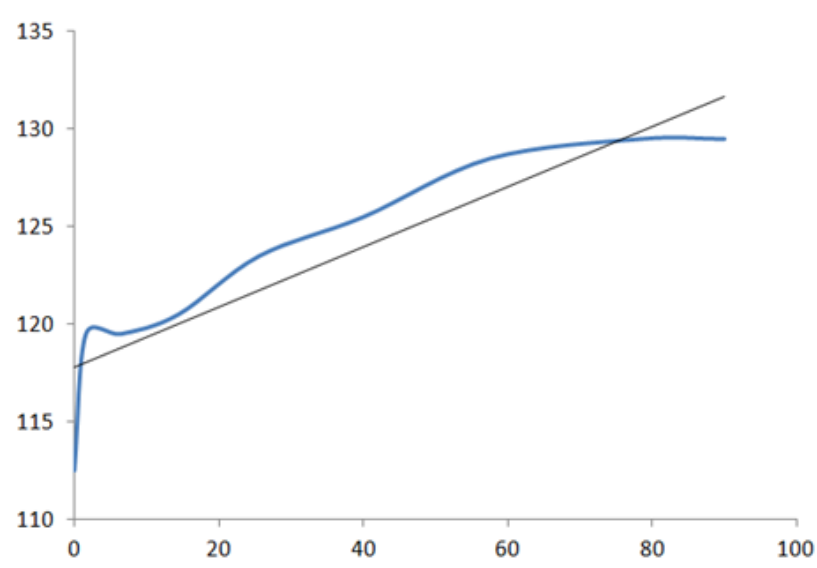

Figure 2. Comparison of the predicted and analytical results base on lippmann equation.

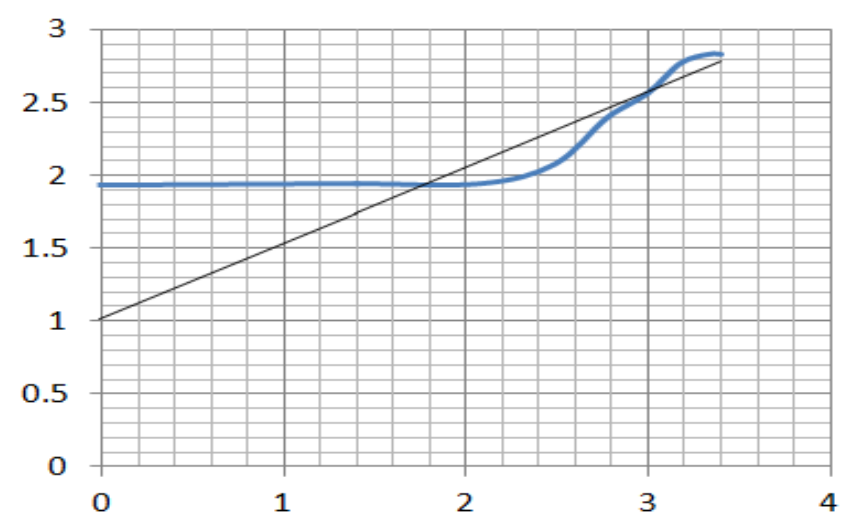

Figure 3. the logarithm of liquid ascent as a function of Logarithm of the Electric Field

\section{References}

[1] E Colgate; H.Matsumoto; J Vac Sci. Technol., (1990), An investigation of ectrowetting- based microstructure J.Voc. Sci. Technol. A8, 3625-3633

[2] Berge, B (1993) Electrocapilarity and wetting of insulator film by water, C.R Acad. Sci. Paris Ser II., (1993), 317, 157-163

[3] Pollack; M.G and Fair R.B.,; A.D Shenderov, (2000), Electrowetting -based actuation of Liquid droplet for microfluidic application, Appl. Phys. let. 77, 1725-1726.

[4] Prins, M.; Welters W.J and Weekamp W.J. (2001), Fluid controlling multichannel structures by electrocapillarity pressure, Science 291, 277-280.

[5] Lienemann J, Greiner A.; Korvink J.G., (2006), Modeling Simulation, and optimization of Electrowetting, IEEE, Tran. Comp-Aided Des Intgr. Cir. Syst., 25, 234-247.

[6] P.Zhou; C.W.Wu, Micro and Nanosystem, (2009), 1, 57-62

[7] X.Shan; H. (1993), Lattice Boltzmann simulating flows with multiple phase and components Phys. Rev. E, 47, 1815-1819

[8] Asberg, A. J, Kponen, A, Merikoski, D and Timnen, J, (2002), lattice Boltzmann simulation of Capillary rise dynamics, J.Stat.Phys 107, 143-153

[9] H.Li; J.Li; Y. Chen; H.Fang, Comput. Phys. (2007),Lattice Boltzmann model simulation of elrctrowetting-on- dielectric in a rough wall channel. Compt. Science, http://arcxiv.org/abs/0708.4182v

[10] Haung,H, Thome D.T, Schaap, M.G and Sukop M.C, (2007), Propsed approximation for contact angles in Shan-and Shentypemulticomponet multiphase lattice Boltzmann models, Phys. Rev E, 76, 066701

[11] Lin, P, (1978), "Some Electrocapiillary-Type Behaviour of Gold Electrode, J. Electrochem. Soc; 194, pp 1077-1098

[12] Kata, E (1991). Electrocapillarity in dielectrics, MacMillan, India Press, Madras.

[13] Chen, J.H and Hsieh, W.H,. (2006), Electrowetting-induced capillary flow in a parallel-plate Channel, J. Colloid. Interface Sci., (2006), 296, 276-283.

[14] Kang K.H.; Kangn I.S and Lee,C.M, (2003), Wetting Tension Due to Coulombic Interaction in Charge- Related Wetting phenomena Langmuir Vol. 19, No. 13, 2003, 548-513. 